\title{
A New Method to Study Goldbach Conjecture
}

\author{
Ke Li \\ Institute of Chemical Industry of Forest Products, CAF, Nanjing, China \\ Email: liketaiping@163.com
}

How to cite this paper: Li, K. (2022) A New Method to Study Goldbach Conjecture. Applied Mathematics, 13, 68-76. https://doi.org/10.4236/am.2022.131006

Received: October 20, 2021

Accepted: January 18, 2022

Published: January 21, 2022

Copyright $\odot 2022$ by author(s) and Scientific Research Publishing Inc. This work is licensed under the Creative Commons Attribution International License (CC BY 4.0).

http://creativecommons.org/licenses/by/4.0/

\begin{abstract}
This paper does not claim to prove the Goldbach conjecture, but it does provide a new way of proof (LiKe sequence); And in detailed introduces the proof process of this method: by indirect transformation, Goldbach conjecture is transformed to prove that, for any odd prime sequence $\left(3,5,7, \cdots, P_{n}\right)$, there must have no LiKe sequence when the terms must be less than $3 \times P_{n}$. This method only studies prime numbers and corresponding composite numbers, replaced the relationship between even numbers and indeterminate prime numbers. In order to illustrate the importance of the idea of transforming the addition problem into the multiplication problem, we take the twin prime conjecture as an example and know there must exist twin primes in the interval $\left[3 P_{n}, P_{n}^{2}\right]$. This idea is very important for the study of Goldbach conjecture and twin prime conjecture. It's worth further study.
\end{abstract}

\section{Keywords}

Goldbach Conjecture, LiKe Sequence, Twin Prime Conjecture, Number Theory

\section{Introduction}

Goldbach conjecture [1] is a mathematical puzzle known all over the world, and has been around for 280 years (1742-2022). At present, the research methods mainly include almost prime, exception set, three prime theorem and almost Goldbach problem. But the latest achievement-the Chen's theorem-has been around for 50 years [2] also. But none of them solved the problem. Why? It's not lack of effort, mainly because we don't find the right way. For this, I find a new way, in contrast to previous methods, it transforms Goldebach conjecture to only study the relationship between primes and composite numbers (Do not need to study the even numbers any more). I named it "LiKe sequence". This method is feasible in theory. This paper briefly introduces the proof process of this method: by indirect transformation, Goldbach conjecture is transformed to prove 
that the terms in LiKe sequence of odd prime sequence $\left(3,5,7, \cdots, P_{n}\right)$ must be greater than $2 \times\left(P_{n+1}-1\right)$. The problem is proved by proving the terms of LiKe sequence $>3 \times P_{n}>2 \times P_{n+1}>2 \times\left(P_{n+1}-1\right)$ step by step. Through this article, we can understand that if we can prove there is no LiKe sequence when the terms are less than $3 \times P_{n}$. For any odd prime sequence $\left(3,5,7, \cdots, P_{n}\right)$, the Goldbach conjecture will be true.

\section{The Definition of LiKe Sequence}

Let $2 N$ represents even numbers and half of it is $N$, all odd primes less than $N$ as the prime sequence $\left(3,5,7, \cdots, P_{n}\right)$, only use these prime factors to represent composite numbers $\left(Y \mid Y \in R, R=3^{x} 5^{y} \cdots P_{n-1}^{i} P_{n}^{j}\right)$. In these composite numbers (Almost composite), if there is a new sequence $\left(Y_{1}, Y_{2}, \cdots, Y_{n}\right)$, and $Y_{n}>Y_{n-1}>\cdots>Y_{2}>Y_{1}$. If the reverse interval of terms is equal to the interval of the terms of the prime sequence (that is $Y_{n}-Y_{n-1}=5-3, Y_{n-1}-Y_{n-2}=7-5$, $\left.\cdots, \quad Y_{2}-Y_{1}=P_{n}-P_{n-1}\right)$. We called $\left(Y_{1}, Y_{2}, \cdots, Y_{n}\right)$ is the corresponding LiKe sequence of the prime sequence $\left(3,5,7, \cdots, P_{n}\right)$. From the Table 1 , it's easy to understand the LiKe sequence, and find that, for any odd prime sequence $\left(3,5,7, \cdots, P_{n}\right)$, there is no LiKe sequence when the terms less than $2 \times\left(P_{n+1}-1\right)$.

\section{Why Is It Equivalent to Goldbach Conjecture?}

As we all known, Golbach conjecture states that all even numbers greater than or equal to 4 (The following even numbers apply to this condition) can be represented as the sum of two prime numbers.

It has an equivalent proposition: For any positive integer $N(\geq 2)$, it's either a prime or there is a number $x$, make both $N-x$ and $N+x$ prime numbers.

That is to say, see Table 2, for any even number $2 N$ (that is $N-x+N+x$ ), all odd primes no greater than $N$ as the prime sequence $\left(3,5,7, \cdots, P_{n}\right)$, there must have a prime number in $\left(2 N-3,2 N-5, \cdots, 2 N-P_{n}\right)$ at least (underline).

Its negative statement is that all $\left(2 N-3,2 N-5, \cdots, 2 N-P_{n}\right)$ are composite numbers.

And, for all composite numbers less than $2 N$, the factors can only be the prime number less than $N$.

Table 1. Prime sequence and the corresponding LiKe sequence.

\begin{tabular}{cccc}
\hline Prime sequence & $(3)$ & $(3,5)$ & $\left(3,5,7, \cdots, P_{n}\right)$ \\
LiKe sequence & $(9),(27), \cdots,\left(3^{n}\right)$ & $(25,27)$ & $\left(Y_{1}, Y_{2}, \cdots, Y_{n}\right)$ \\
& & $25=5^{2} ;$ & $Y_{n}=3^{x} 5^{y} \cdots P_{n-1}^{i} P_{n}^{j} ;$ \\
Character & $3^{n}$ & $27=3^{3}$, & $Y_{n}-Y_{n-1}=5-3, \cdots$, \\
& & $27-25=5-3$ & $Y_{2}-Y_{1}=P_{n}-P_{n-1}$ \\
Deduction & $9>2 \times(5-1)$ & $25>2 \times(7-1)$ & $Y_{1}>2 \times\left(P_{n+1}-1\right)$ \\
\hline
\end{tabular}


Table 2. LiKe matrix.

\begin{tabular}{|c|c|c|c|c|c|c|c|c|}
\hline $2 N$ & \multicolumn{8}{|c|}{$2 N-P_{n}$} \\
\hline 6 & $\underline{3}$ & 0 & 0 & 0 & 0 & 0 & 0 & 0 \\
\hline 8 & $\underline{5}$ & 0 & 0 & 0 & 0 & 0 & 0 & 0 \\
\hline 10 & $\underline{7}$ & $\underline{5}$ & 0 & 0 & 0 & 0 & 0 & 0 \\
\hline 12 & 9 & $\underline{7}$ & 0 & 0 & 0 & 0 & 0 & 0 \\
\hline 14 & $\underline{11}$ & 9 & $\underline{7}$ & 0 & 0 & 0 & 0 & 0 \\
\hline 16 & $\underline{13}$ & $\underline{11}$ & 9 & 0 & 0 & 0 & 0 & 0 \\
\hline 18 & 15 & $\underline{13}$ & $\underline{11}$ & 0 & 0 & 0 & 0 & 0 \\
\hline 20 & $\underline{17}$ & 15 & $\underline{13}$ & 0 & 0 & 0 & 0 & 0 \\
\hline 22 & $\underline{19}$ & $\underline{17}$ & 15 & $\underline{11}$ & 0 & 0 & 0 & 0 \\
\hline 24 & 21 & $\underline{19}$ & $\underline{17}$ & $\underline{13}$ & 0 & 0 & 0 & 0 \\
\hline 26 & $\underline{23}$ & 21 & $\underline{19}$ & 15 & $\underline{13}$ & 0 & 0 & 0 \\
\hline 28 & 25 & $\underline{23}$ & 21 & $\underline{17}$ & 15 & 0 & 0 & 0 \\
\hline 30 & 27 & 25 & $\underline{23}$ & $\underline{19}$ & $\underline{17}$ & 0 & 0 & 0 \\
\hline 32 & $\underline{29}$ & 27 & 25 & 21 & $\underline{19}$ & 0 & 0 & 0 \\
\hline 34 & $\underline{31}$ & $\underline{29}$ & 27 & $\underline{23}$ & 21 & $\underline{17}$ & 0 & 0 \\
\hline 36 & 33 & $\underline{31}$ & $\underline{29}$ & 25 & $\underline{23}$ & $\underline{19}$ & 0 & 0 \\
\hline 38 & 35 & 33 & $\underline{31}$ & 27 & 25 & 21 & $\underline{19}$ & 0 \\
\hline 40 & $\underline{37}$ & 35 & 33 & $\underline{29}$ & 27 & $\underline{23}$ & 21 & 0 \\
\hline$\ldots$ & $\mathrm{O}$ & $\mathrm{O}-2$ & $\ldots$ & O-(11-3) & $\ldots$ & $\ldots$ & $\ldots$ & $\ldots$ \\
\hline
\end{tabular}

So, for all the prime sequence $\left(3,5,7, \cdots, P_{n}\right)$, if there is no LiKe sequence when the terms must less than $2 \times\left(P_{n+1}-1\right)$, the Goldbach conjecture will be true.

\section{What Is the Proof Path?}

To sum up, the method must be right, but how do we prove Goldbach conjecture in this way? We have two paths:

A: If when $2 N$ is greater than a certain number, there is no LiKe sequence for all odd prime numbers before $N$, and make sure the numbers in $2 N$ match the Goldbach conjecture, it can be proved directly.

B: If there is a LiKe sequence, the range of LiKe sequence should be determined. If it can be proved that the terms of the LiKe sequence must be greater than $2 N$ (there is no LiKe sequence less than $2 N$ ), it can also be proved.

For path A, we need only determine a prime number, such as 7 (This is just one example), make sure that the numbers up to 20 (that is $2 \times\left(P_{n+1}-1\right)$ ) conform to Goldbach conjecture; And $\left(3,5, \cdots, P_{n}\right)$ has no LiKe sequence $\left(P_{n} \geq 7\right)$. 
The Goldbach conjecture will be proved. It's a good idea, but obviously it might be very hard (It's may be an entirely new conjecture), and we don't have to. For Goldbach conjecture, we just need to finish proving B. Compared with path A, it limits the scope and greatly reduces the difficulty.

Here are some of my simple derivations for your reference.

\section{Theorem 1:}

Given the odd primes sequence $\left(3,5,7, \cdots, P_{n}\right)$, and the terms of its corresponding LiKe sequence can only be $>2\left(P_{n+1}-1\right)$.

Proof:

To prove this theorem, we must know the following two lemmas:

Lemma 1:

The terms in minimum LiKe sequence of odd prime sequence $\left(3,5,7, \cdots, P_{n}\right)$ is greater than $3 \times P_{n}$.

The LiKe sequence of (3) are (9), (27), .., $\left(3^{n}\right)$. They all $\geq 3 \times 3$;

The smallest LiKe sequence of $(3,5)$ is $(25,27)$. And $25>3 \times 5$.

For all the prime sequence $\left(3,5, \cdots, P_{n}\right)$, see Table 3 , we can easily verify that there is no LiKe sequence when the terms must less than $3 \times P_{n}$. But how prove it? The proof is as follows:

Really the sequence of $(3,5,7)$ or $(3,5,7,11)$ not only no LiKe sequence less than $3 \times P_{n}$ but also no LiKe sequence, the proof is easy:

For (3)

It has infinite numbers of LiKe sequence, and the smallest is 9 .

So (3) has no LiKe sequence when the terms less than $3 \times 3$.

For $(3,5)$

Obviously $3 \times 5$ is the smallest new composite number.

And since you can't have a composite of 3 with a difference of 2 .

So $(3,5)$ has no LiKe sequence when the terms less than $3 \times 5$.

But the proof that $(3,5)$ has LiKe sequences helps the theorem. The proof is as follows:

Because the odd composite difference of 3 is $2 \times 3>5-3$.

And the odd composites difference of 5 is $2 \times 5>5-3$.

So if $(3,5)$ have the LiKe sequences, it can only be a permutation of $\left(3^{i}, 5^{j}\right)$.

Because:

$$
3^{i} \bmod 9=0
$$

Table 3. Odd prime sequence and LiKe sequence.

\begin{tabular}{cccc}
\hline Prime sequence & Almost composite & Smallest LiKe sequence & range \\
\hline$(3)$ & $9,27,81, \ldots, 3^{i}$ & $(9)$ & $\geq 9$ \\
$(3,5)$ & $9,15,25,27,45, \ldots$ & $(25,27)$ & $>15$ \\
$(3,5,7)$ & $9,15,21,25,27,35,45, \ldots$ & - & $>21$ \\
$(3,5,7,11)$ & $9,15,21,25,27,33,35,45, \ldots$ & - & $>33$ \\
$\left(3,5,7,11, \ldots, P_{n}\right)$ & $9,15,21,25,27,33,35,39,45, \ldots$ & - & $>3 \times P_{n}$ \\
\hline
\end{tabular}


And:

$5^{j} \bmod 9 \in\{7,8,4,2,1,5\}$ contains 2 .

So the permutation of $\left(3^{i}, 5^{j}\right)$ can form the LiKe sequences of $(3,5)$.

$(25,27)$ is fine.

But it's obviously $>15$.

The proof is very clever, we can do the same thing with the proof of $\left(3,5, \cdots, P_{n}\right)$.

For $(3,5,7)$

Let $(a, b, c)$ is a LiKe sequence of $(3,5,7)$.

Similarly:

$$
2 \times 3>7-3 ; 2 \times 5>7-3 ; 2 \times 7>7-3
$$

So $(a, b, c)$ can only be a permutation of $\left(3^{i}, 5^{j}, 7^{k}\right)$

Because:

$$
7^{2}>3 \times 7
$$

So $(3,5,7)$ has no LiKe sequence when the terms less than $3 \times 7$.

Of course, $(3,5,7)$ actually has no corresponding LiKe sequence. The proof is simple:

Because:

$$
5^{j} \bmod 25=0
$$

And:

$$
7^{k} \bmod 25 \in\{24,18,1,7\}
$$

But LiKe sequence requires:

$$
\left|7^{k}-5^{j}\right| \in\{2,4\} \not \subset\{24,18,1,7\}
$$

it's impossible.

So $(3,5,7)$ has no LiKe sequence.

For $(3,5,7,11)$

Let $(a, b, c, d)$ is a LiKe sequence of $(3,5,7,11)$, and $a<b<c<d$.

Similarly:

$$
2 \times 5>11-3 ; 2 \times 7>11-3 ; 2 \times 11>11-3
$$

So as factors 5, 7, 11 can occur at most once in $(a, b, c, d)$.

And:

$$
2 \times 3<11-3 ; 2 \times 2 \times 3>11-3
$$

So the factor 3 can occur up to two times in the $(a, b, c, d)$.

Suppose $m$ is the greatest common divisor of $a$ and $b$.

$m$ is divisible by at least one of $3,5,7$ and 11 .

That is $b-a$ can be divisible by $m$.

But according to the LiKe sequence: $b-a=11-7=4$, excluding factors 3,5 , 7 and 11.

So $a$ and $b$ have no common divisor.

Similarly: $b$ and $c, c$ and $d ; b$ and $d$; $a$ and $d$ have no common divisor.

But $c-a=11-5=6$, It's divisible by 3 . 
So $a$ and $c$ can have a common factor of 3 .

Suppose $a$ and $c$ have a common factor of 3 .

Because in addition to $a$ and $c$, the other terms are pairwise coprime.

So between $a$ and $c$, one of them has to be $3^{i}$, the other's factor is 3 and one of $5,7,11$; The factors of $b$ and $d$, can only be one of the two remaining primes. (That is only one of $a$ and $c$ can have two factors)

Because:

$$
5^{\mathrm{i}} \bmod 25=0
$$

And:

$7^{i} \bmod 25 \in\{24,18,1,7\}$, does not contain 2,4 and 8 .

$11^{i} \bmod 25 \in\{21,6,16,1,11\}$, it also does not contain 2,4 and 8 .

So $b$ and $d$ has no factor of 5 .

That is one of $a$ and $c$ must be $3^{i}$ and the other must be $3^{i} 5, b$ and $d$ are each $7^{k}$ and $11^{m}$.

Also because:

$11^{i} \bmod 49 \in\{23,8,39,37,15,18,2\}$, does not contain 4 .

And LiKe sequence requirements:

$$
d-b=\left|11^{m}-7^{k}\right|=4 \notin\{23,8,39,37,15,18,2\}
$$

So $a$ and $c$ have no common divisor.

So $a, b, c$ and $d$ must prime to each other, and they can only be a permutation of $\left(3^{i}, 5^{j}, 7^{k}, 11^{m}\right)$.

Obviously:

$$
11^{2}>3 \times 11
$$

So $(3,5,7,11)$ has no LiKe sequence when the terms less than $3 \times 11$.

In the same way:

$$
\left|7^{k}-5^{j}\right| \in\{2,4,6,8\} \not \subset\{24,18,1,7\}
$$

So $(3,5,7,11)$ has no LiKe sequence.

As mentioned above, it is not necessary to prove that there is no LiKe sequence.

We only need to prove there is no LiKe sequence when the terms less than $3 \times P_{n}$.

For $(3,5,7,11,13)$

Because $(3,5,7,11)$ has no LiKe sequence less than $3 \times 11$.

When add a prime number 13 .

It's not hard to prove that the LiKe sequence of $(3,5,7,11,13)$ can only be a permutation of $\left(3^{i}, 5^{j}, 7^{k}, 11^{m}, 13^{n}\right)$.

And:

$$
13^{2}>3 \times 13
$$

So there is no LiKe sequence of $(3,5,7,11,13)$ when the terms less than $3 \times P_{n}$. 
For $\left(3,5,7,11, \cdots, P_{n-1}, P_{n}\right)$

If $\left(3,5,7,11, \cdots, P_{n-1}\right)$ has no LiKe sequence when the terms less than $3 \times P_{n-1}$.

Similarly, it's not hard to prove there is no LiKe sequence of

$\left(3,5,7,11, \cdots, P_{n-1}, P_{n}\right)$ when the terms less than $3 \times P_{n}$.

So for odd prime sequence $\left(3,5, \cdots, P_{n}\right)$, if has LiKe sequence, the terms of it must greater than $3 \times P_{n}$.

Flexible turn addition problems into multiplication problems can often be clever to solve the problem, such as this millennium puzzle-there are infinitely many prime $P$ make $P+2$ is also a prime [3]. By transforming, making the problem easier to understand, see the theorem 2.

\section{Theorem 2:}

There are infinitely many odd composite numbers with a distance of 6 , and no odd composite numbers between them.

The odd composite numbers with factor of 3 (mark them as $3 C$, the same below) are:

$$
3 C=3 \times(3,5,7,9, \cdots)=(9,15,21,27,33,39, \cdots)
$$

The distance is 6 (make it as VI for convenience), and there's an infinitely many VI. Take these VI as a benchmark, there are two main reasons.

1) There are two odd numbers inside every VI;

2) If they are not composite numbers (not be split up), they must be twin prime numbers.

Then investigate its splitting by odd composite numbers of other prime numbers:

The odd composite numbers with factor of 5

$$
5 C=5 \times(3,5,7,9, \cdots)=(15,25,35,45,55, \cdots)
$$

The odd composite numbers with factor of 7

$$
\begin{gathered}
7 C=7 \times(3,5,7,9, \cdots)=(21,35,49,63,77, \cdots) \\
\ldots \\
P_{n} C=P_{n} \times(3,5,7,9, \cdots)
\end{gathered}
$$

It's not hard to see, when add to $P_{n} C$ each split starts at $P_{n}^{2}$. So we can prove there must have VI in the interval $\left[P_{n}^{2}, P_{n+1}^{2}\right]$ couldn't be split up. It's almost Brocard conjecture, it's very hard.

In addition, the period of $3 C$ is $2 \times 3$; when add to $5 C$, the period becomes $\prod_{1}^{3} P_{3}$; and when add to $P_{n} C$, the period is $\prod_{1}^{n} P_{n}$. So we can prove there always have VI in the interval $\left[3 P_{n}, 3 P_{n}+\prod_{1}^{n} P_{n}\right]$ couldn't be split up. But it's too big $\left(3 P_{n}+\prod_{1}^{n} P_{n}>P_{n+1}^{2}\right.$ when $\left.P_{n}>5\right)$.

Fortunately, we can prove there must have VI in the interval $\left[3 P_{n}, P_{n}^{2}\right]$ couldn't be split up.

The distance of $3 C$ are all $6(\mathrm{VI})$;

When add to $5 C$, the distance of $5 C$ is $2 \times 5$, and every 10 crosses a VI, and the split starts at $5^{2}$. So the VI in the interval $\left[3 \times 5,5^{2}\right]$ couldn't be split up;

When add to $7 C, 3 \times 7$ is just one less VI than $3 \times 5$, but $7^{2}$ is a lot more VI 
than $5^{2}$, and the split starts at $7^{2}$; the distance $(2 \times 7)$ is bigger. So there must left VI in the interval $\left[3 \times 7,7^{2}\right]$ couldn't be split up;

Similarly, there must have VI in the interval $\left[3 P_{n}, P_{n}^{2}\right]$ couldn't be split up $(n \geq 3)$. And there's always a $P_{m}$ make $3 P_{m}>P_{m}^{2}$ and there also have VI in the interval $\left[3 P_{m}, P_{m}^{2}\right]$ couldn't be split up.

And there are infinitely many primes, so there must be infinity many VI couldn't be split up, and these odd numbers inside VI are twin primes. $\square$

So this idea is very important. Let's get to the point, see Lemma 2.

Lemma 2:

When $N$ is large enough, there must be primes between $N$ and $1.5 N$.

It involves an old classic problem (Is there exists a prime number in the interval $[k n,(k+1) n]$ ?) and obviously true, see ref. [4].

In fact, so far the old problem has been proved there is at least one prime in the interval [3n, 4n] and [4n, 5n] [5] [6].

So when $N$ is large enough, there must be primes between $N$ and $1.5 N$. $\square$

With the above two lemmas, we can prove theorem 1, as follows:

From the lemma 2, we can get:

When $N$ is large enough, there must be:

$$
\left(3 \times P_{n}\right) /\left(2 \times P_{n+1}\right)>1
$$

that is:

$$
3 \times P_{n}>2 \times P_{n+1}
$$

And because:

$$
2 \times P_{n+1}>2 \times\left(P_{n+1}-1\right)
$$

So:

$$
3 \times P_{n}>2 \times\left(P_{n+1}-1\right)
$$

From the lemma 1, we can get:

The terms of LiKe sequence $>3 \times P_{n}$

So:

$$
\text { The terms of LiKe sequence }>2 \times\left(P_{n+1}-1\right)
$$

So for sufficiently large numbers, the theorem 1 is right and the Goldbach conjecture is true too.

Q.E.D

\section{Conclusion}

To sum up, the new method (LiKe sequence) not only can solve the Goldbach conjecture, but only needs to prove for any odd prime sequence $\left(3,5,7, \cdots, P_{n}\right)$, there must have no LiKe sequence when the terms are less than $3 \times P_{n}$, the Goldbach conjecture will be true. The biggest benefit of this method is only to study prime numbers and composite numbers (Only study the property of multiplication); no longer need to study the relationship between even numbers and 
prime numbers (The relation between addition and multiplication). In order to illustrate the importance of the idea of transforming the addition problem into the multiplication problem, we take the twin prime conjecture for example and know that if there exists twin primes in the interval $\left[3 P_{n}, P_{n}^{2}\right]$ it will be true. This idea provides a convenient way to study Goldbach conjecture, twin prime conjecture and other number theory problems, so it's very important. Moreover, lemma 1 will be a new classical problem without considering the scope (don't have to less than $\left.3 \times P_{n}\right)$ : Does there exist a LiKe sequence for any odd prime sequence $\left(3,5,7, \cdots, P_{n}\right)$ when $P_{n} \geq 7$ ? Of course, as the article has shown, the odd prime sequence $(3,5,7)$ and $(3,5,7,11)$ have no LiKe sequence. I think this question will catch on soon after this article.

\section{Conflicts of Interest}

The author declares no conflicts of interest regarding the publication of this paper.

\section{References}

[1] Wang, Y. (2002) The Goldbach Conjecture. World Scientific Publishing Co., Singapore, 402-408.

[2] Chen, J.R. (1978) On the Representation of a Large Even Integer as the Sum of a Prime and the Product of at Most Two Primes. Science China Mathematic, 21, 421-430.

[3] Zhang, Y.T. (2014) Bounded Gaps between Primes. Annals of Mathematics, 179, 1121-1174. https://doi.org/10.4007/annals.2014.179.3.7

[4] El Bachraoui, M. (2006) Prime in the Interval [2n, 3n]. International Journal of Contemporary Mathematical Sciences, 1, 617-621. https://doi.org/10.12988/ijcms.2006.06065

[5] Loo, A. (2011) On the Primes in the Interval [3n, 4n]. International Journal of Applied Mathematics and Computer Science, 6, 1871-1882.

[6] Balliet, K.D. (2015) On the Prime Numbers in the Interval [4n, 5n]. arXiv Preprint. https://arxiv.org/pdf/1511.04571.pdf 